\title{
Magnetic-field-induced changes of the isotropic-nematic phase transition in side-chain polymer liquid crystals
}

\author{
M. I. Boamfă, ${ }^{1, *}$ K. Viertler, ${ }^{2}$ A. Wewerka, ${ }^{2}$ F. Stelzer, ${ }^{2}$ P. C. M. Christianen, ${ }^{1}$ and J. C. Maan ${ }^{1}$ \\ ${ }^{1}$ Research Institute for Materials, High Field Magnet Laboratory, University of Nijmegen, Toernooiveld 1, 6525 ED Nijmegen, \\ The Netherlands \\ ${ }^{2}$ Institute for Chemistry and Technology of Organic Materials, Graz, University of Technology, Stremayrgasse 16/1, \\ A-8010 Graz, Austria
}

(Received 14 August 2002; published 15 May 2003)

\begin{abstract}
The isotropic-nematic $(I-N)$ phase transition of side-chain polymer liquid crystals is intrinsically a weak first-order transition with a biphasic region spread over a wide temperature interval. In the presence of high magnetic fields we find the $I-N$ transition to become a strong first order. The $I-N$ biphasic region shrinks its temperature window as larger magnetic fields are applied, until it completely disappears and the transition completes at a fixed temperature. We interpret this behavior as a consequence of the nonlinear coupling of the magnetic field to the system free energy, via the suppression of the order fluctuations in the nematic mesophase at the $I-N$ transition crossing.
\end{abstract}

DOI: 10.1103/PhysRevE.67.050701

Side-chain polymer liquid crystals, SCPLCs, are made of rigid mesogenic units attached laterally to a polymeric chain via a flexible spacer, which gives the mesogenes enough freedom to form Liquid Crystalline mesophases [1-3]. The SCPLCs phase space is characterized by a glass phase at low temperatures followed by (an) intermediate ordered mesophase(s) and an isotropic phase at high temperatures. The SCPLCs phase transitions are very broad, taking place over a wide temperature region, where a mesophase coexists with either the isotropic melt or another mesophase-behavior generally attributed to a certain degree of polydispersity [4].

Combining the properties of polymers with the selforganization of liquid crystals, SCPLCs are of fundamental research interest [2-4] as well of importance for many applications [5] such as optically nonlinear devices [6], displays $[7,8]$, and optical data storage [9]. The isotropicnematic phase $I-N$ transition is a key point in understanding the properties of these hybrids and is as well a central feature in any method that deals with SCPCLs processing [10-13].

Here we report a study of the $I-N$ transition of SCPLCs in high magnetic fields. We used two thermotropic nematic SCPLCs: a polynorbornene and a polyacrylate-derivative SCPLC, both with cyanobiphenyl groups as mesogenes and methylene units as spacers, see inset Fig. 1(b). We studied the polynorbornene SCPLCs in two distinct conformations, with a spacer length of 5 methylene units and respective 3 methylene units: Poly $\left(( \pm)\right.$-exo,endo-bis $\left\{5-\left(\left(4^{\prime}\right.\right.\right.$-cyanobiphenyl-4yl)oxy)pentyl\}norborn-5-ene-2,3-dicarboxylate) [14], referred as $C 5$ and Poly $\left(( \pm)\right.$-exo,endo-bis $\left\{3-\left(\left(4^{\prime}\right.\right.\right.$-cyanobiphenyl-4-yl)oxy)propyl\}norborn-5-ene-2,3-dicarboxylate) referred as C3 [14]. The polyacrylate-derivative: Poly$\left\{4-\left(\left(4^{\prime}\right.\right.\right.$-cyanobiphenyl-4-yl)oxy)butyl $\} a c r y l a t e$, referred to as $B 4$, has a more flexible backbone chain in comparison with the polynorbornene one and its spacer is 4 methylene units long. The samples have been studied for different polymerization degrees, $n$. The samples were prepared as films on

\footnotetext{
*Email address: boamfa@sci.kun.nl
}

PACS number(s): 64.70.Md, 61.30.Gd, 61.30.Vx

optical glass support by spin coating from solution and subsequent solvent removal by annealing. For experimental investigation, the sample is placed in a temperature-controlled chamber, within the bore of a $20 \mathrm{~T}$ resistive "Bitter" magnet. The magnetic-field-induced birefringence and the optical transmission are simultaneously measured, using a polarization-intensity double modulated laser [15]. The bire-
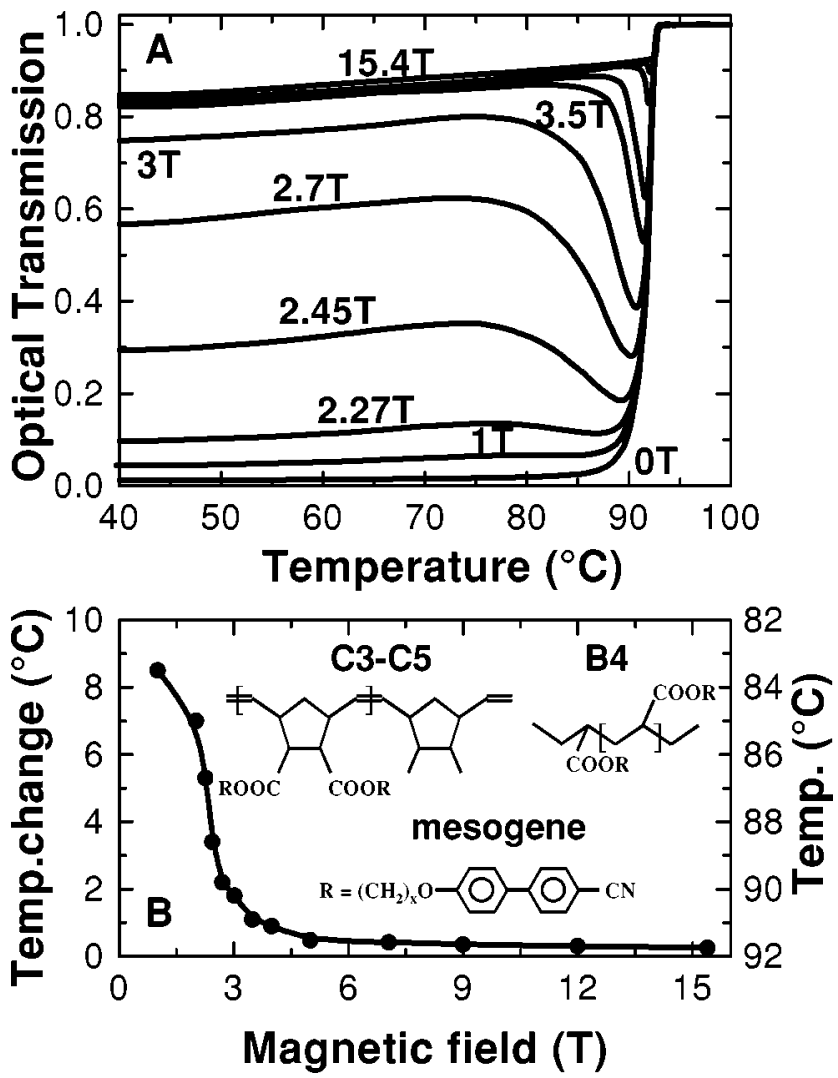

FIG. 1. (a) Optical transmission evolution of a $B 4$ sample, $n$ $=30,20 \mu \mathrm{m}$ film, cooled in different magnetic fields. (b) Changes of the optical transmission turning point, extracted from the upper panel curves. Inset (a) chemical formulas for $C 3(x=3), C 5(x$ $=5)$, and $B 4(x=4)$. 
fringence signal is indicative of the macroscopic order of the sample [16]. In the nematic phase the SCPLCs features a microscopic texture of locally ordered domains, randomly oriented to each other, which give rise to light scattering. At the probing wavelength, He-Ne, $543.5 \mathrm{~nm}$, the samples do not absorb and the scattered light is the only contribution to the optical transmission, allowing direct monitoring of the sample domain morphology. All experiments were done at $10^{\circ} \mathrm{C} / \mathrm{min}$ cooling-warming rate, which was verified to be sufficiently slow.

Figure 1(a) presents the optical transmission evolution of a $B 4, n=30$, sample, cooled in different magnetic fields. In zero magnetic field, from high transmission in the isotropic phase the sample starts to scatter light as it undergoes the $I-N$ transition; the transmission constantly decreases during the biphasic region and the sample ends up completely opaque deep in the nematic phase. For low applied magnetic fields, the induced changes are small and the sample follows almost the same path as the cooled one in a zero magnetic field. In stronger magnetic fields, there is a point in the biphasic region where the transmission completely changes its trend, showing a sharp increase, followed by saturation at an intermediate value in the nematic phase. Higher applied magnetic fields move the transmission turning point toward the beginning of the $I-N$ transition, $T_{I N}$, while the transmission saturates at higher values. For fields above $7 \mathrm{~T}$ the biphasic region completely disappears and the transition takes place in a very narrow interval of $0.5^{\circ} \mathrm{C}$. The transmission turning point evolution, Fig. 1(b), shows a strong onset around $2.5 \mathrm{~T}$, followed by saturation for fields above $7 \mathrm{~T}$. For a $B 4$ sample with a lower polymerization degree, $n=20$, a similar effect is observed, in this case the biphasic region disappears around 5.5 T. For a $C 5$ sample with $n=20$, the results are analog. Significant effects start being observable at about $5 \mathrm{~T}$ and fields about $17 \mathrm{~T}$ are needed to completely close the biphasic region. A $C 5$ with $n=30$, shows a comparable behavior. A significant effect appears around $8 \mathrm{~T}$ but the maximum available field $20 \mathrm{~T}$ only partially closes the biphasic region. For a $C 5$ sample with an even higher polymerization degree, $n=40$, an effect starts being visible around $18 \mathrm{~T}$, while until $20 \mathrm{~T}$ we observe only a modest narrowing of the biphasic region. The $C 3$ samples did not show an effect in the maximum applied field of $20 \mathrm{~T}$ for any of the investigated polymerization degrees: $n=20, n=30$, $n=40$.

Figure 2 presents the birefringence signal and the optical transmission of a $C 5$ sample recorded upon cooling in $12 \mathrm{~T}$. The birefringence clearly shows the pretransitional ordering followed by a sharp jump in a narrow interval of about $0.5^{\circ} \mathrm{C}$. The curve continues with a smooth increase throughout the biphasic region, followed by saturation as it approaches the glass phase. In the pretransitional region the transmission features a small decrease followed by an abrupt decrease starting at $T_{I-N}$, which extends for the next approximately $0.5^{\circ} \mathrm{C}$. From here on, the transmission changes its trend and continuously increases until the end of the biphasic region. In the nematic phase the transmission signal remains constant, indicating that the ordering, as revealed by the birefringence, affects uniformly the sample without creating

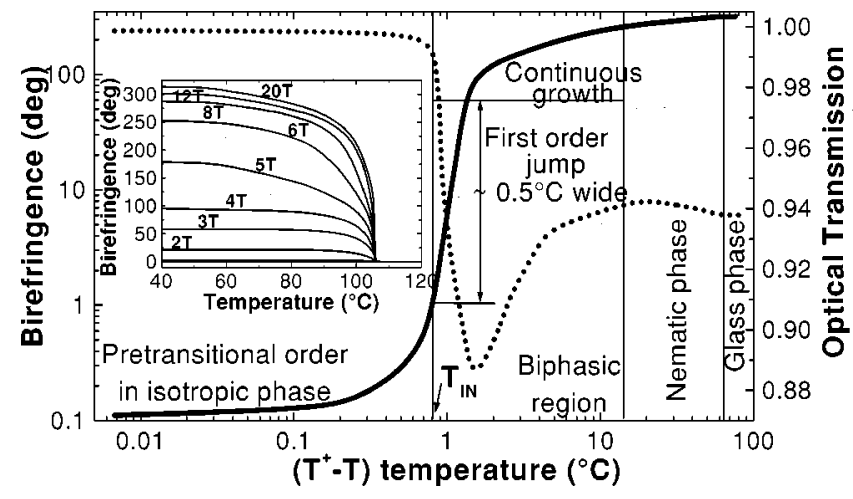

FIG. 2. Birefringence (full line) and optical transmission (dotted line) of a $C 5$ sample, $n=20,10 \mu \mathrm{m}$ film, simultaneously recorded during cooling in $12 \mathrm{~T}$, versus $\left(T^{+}-T\right)$ temperature scale, with $T^{+} \approx T_{I-N}+1{ }^{\circ} \mathrm{C}$. Inset: magnetic-field-induced birefringence of the same $C 5$ sample, recorded upon cooling in different fields.

more scattering. The inset presents the birefringence of the same sample as cooled in different fields. The onset of the birefringence always starts at $T_{I-N}$ and saturates toward the nematic-glass transition, but the saturation values are higher for larger applied fields.

For a better understanding of these observations, let us first consider the $I-N$ transition dynamics in the absence or presence of an applied magnetic field. Intrinsically, the $I-N$ transition of SCPLCs is a two-step process. A volume fraction of the sample undergoes a sharp first-order jump at a fixed temperature, $T_{I-N}$, via nucleation of nematic islands at equilibrium with the surrounding isotropic phase. The different refractive indices between the nematic islands and the adjacent isotropic component cause strong light scattering (Fig. 1). The rest volume fraction of the sample changes continuously from isotropic to nematic over a large temperature range, via a growth mechanism on the already existing nematic nuclei, Fig. 3. This particular behavior originates in the fact that the rotational orientation of the mesogenes is hindered by the polymer backbones [17]. In the absence of an applied external field, the directors of the nucleated nematic islands are randomly oriented and the particular orientation of each individual nematic island is fixed by the mesogene-backbone coupling. Therefore, as is pictorially sketched in Fig. 3, any configuration of the isotropic component to become nematic between two neighboring nematic islands costs an extra elastic energy, see also Eq. (1). To overcome this energetic barrier, a fraction of the sample undergoes suppercooling, impending the transition to be consumed at a fixed temperature. At the end of the biphasic region the sample volume become mostly covered by nematic islands with a different orientation relative to each other, which evolves further into nematic domains separated by sharp domain walls; Fig. 3, upper panel. This causes a further increase in the scattered light, Fig. 1, while the birefringence of this multidomain morphology is very small due to the absence of a common director orientation, Fig. 2 inset.

In the presence of an applied magnetic field, as $T_{I-N}$ is reached, nematic islands nucleate and in the same time undergo orientation along the magnetic-field lines. This ordering results in a strong suppression of the elastic energetic 
No magnetic field applied
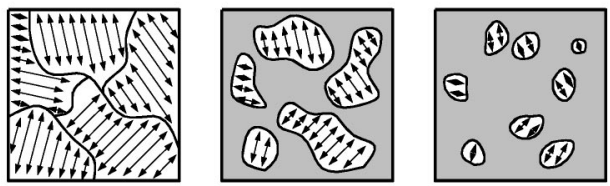

Nematic

Biphasic region

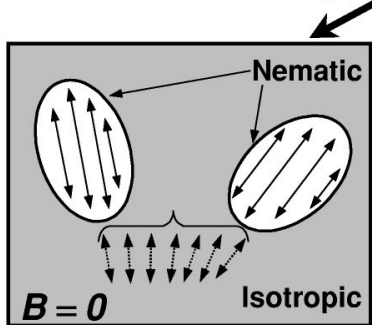

Costs extra elastic energy

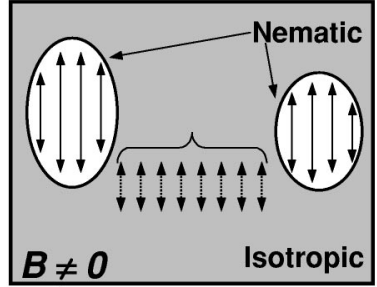

Nematic

Biphasic region

Costs no elastic energy
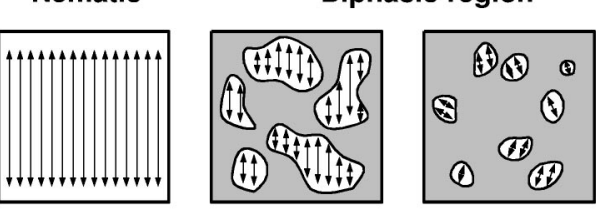

In magnetic field

FIG. 3. Schematic representation of the biphasic region where nematic nuclei coexist at equilibrium with the surrounding isotropic component, in the absence (left and upper panel) [presence (right and lower panel)] of an applied magnetic field. The central panels illustrate the possible configuration of the isotropic component to become nematic (dotted arrows) and its energetic costs.

costs and, as a consequence, a larger volume fraction of the sample turns nematic at $T_{I-N}$, Fig. 3 , with the biphasic region shrinking accordingly. The magnetic field leaves unchanged the local order, because the magnetic energy per molecule is small compared to the thermal fluctuations, but changes the orientation of the nematic islands as a whole, affecting only the director order. At the end of the biphasic region the individual directors are aligned along the magnetic field, causing the system to evolve into a monodomain morphology, Fig. 3, (lower panel), which correspond to a high transmission (Fig. 1) and large birefringence (Fig. 2 inset).

Following a Rayleigh-Debye approach [18], we have studied theoretically the light scattering of the SCPLCs in the absence and presence of a magnetic field [15]. We have treated the nematic islands of domains as scattering centers, with their orientations relative to the magnetic field described by a statistical orientation function [16]. In our model the scattering occurs due to differences in the refractive indices between the nematic islands and the surrounding isotropic phase or due to different orientation of the optical axis in neighboring nematic domains. We found two distinct scattering regimes: the beginning of the transition, with isotropic and nematic components with different refractive indices, and the end of the transition, with only nematic domains with the same refractive index but different projections. In the absence of induced order, the $I-N$ transmission appears as a monotonic decrease of the optical trans- mission, with no discontinuities due to the crossover from one regime to the other. This is consistent with the experimental observations, see the $0 \mathrm{~T}$ curve in Fig. 1(a). In the presence of an applied magnetic field we find that the isotropic-nematic islands regime is dominated by the existence of two different refractive indices, whereas the fieldinduced orientation of the nematic islands changes little the overall scattering. However, in the all-nematic regime, the scattering strongly depends by the magnetic-field-induced orientation, with the limit of no scattering in the case of total alignment. It is only at the crossover between the two regimes that a turn in transmission can occur. Optical microscopy analysis consistently confirmed this theoretical observation.

Consequently it is correct to associate the experimentally observed transmission turning point, Fig. 1 and Fig. 2, with a point within the biphasic region, with its position scaling relative to the temperature window of the biphasic region.

To analyze these observations we apply the Landau-de Gennes (LDG) free energy expansion [19-21] to the specific case of SCPLCs in magnetic field:

$$
\begin{aligned}
F= & a\left(T-T^{*}\right) Q^{2}-B_{c t} Q^{3}+C Q^{4}+\Delta \chi B^{2} Q \\
& +L(d Q / d r)^{2}+K_{m-b} Q,
\end{aligned}
$$

where $Q, T$, and $T^{*}$ are the uniaxial nematic order parameter, temperature, and the minimum supercooling temperature. The expansion coefficients $a, B_{c t}, C$, are constants related to the SCPLCs properties. $L$ is related to the elastic constants [21], $K_{m-b}$ is the mesogene-backbone coupling energy, $\Delta \chi$ is the anisotropy of the mesogene diamagnetic, susceptibility and $B$ is the magnetic-field induction.

The LDG framework [19-21] predicts that as the value of the applied magnetic field increases, the transition temperature moves to higher values while the order of the transition changes toward second order. Pretransitional magnetic-field ordering weakens the order parameter jump at the transition and there is a critical point where the transition becomes second-order and for higher fields the transition completely disappear. However, typical numbers show that the possibility of such an experimental observation is quite remote, since it requires magnetic fields of the order $10^{3}-10^{4} \mathrm{~T}$. Because the typical fields used for our experiments are $B \leqslant 20 \mathrm{~T}$, we neglect the direct diamagnetic contribution term $\Delta \chi B^{2} Q$. The mesogene-backbone coupling term $K_{m-b} Q$ is comparable with the magnetic one [17] and we neglect it as well. However, both terms are indirectly present in the order fluctuation term $L(d Q / d r)^{2}$ : one responsible for the its nonvanishing character and one for its value. Making use of the factorization: $L(d Q / d r)^{2}=L Q^{2}\left(A_{D} / \xi\right)^{2}$ and of $\xi$ $=\left[L / a\left(T-T^{*}\right)\right]^{1 / 2}$, Ref. [21], one finds the free energy to have the following expression:

$$
F=a\left(T-T^{*}\right) Q^{2}\left(1+A_{D}^{2}\right)-B_{c t} Q^{3}+C Q^{4},
$$

where $A_{D}$ is the amplitude of the director order fluctuations and $\xi$ is the fluctuation correlation length. Approaching the $I-N$ transition from above, the $A_{D}$ term makes the transition to have a weak first-order character with a biphasic isotropic- 


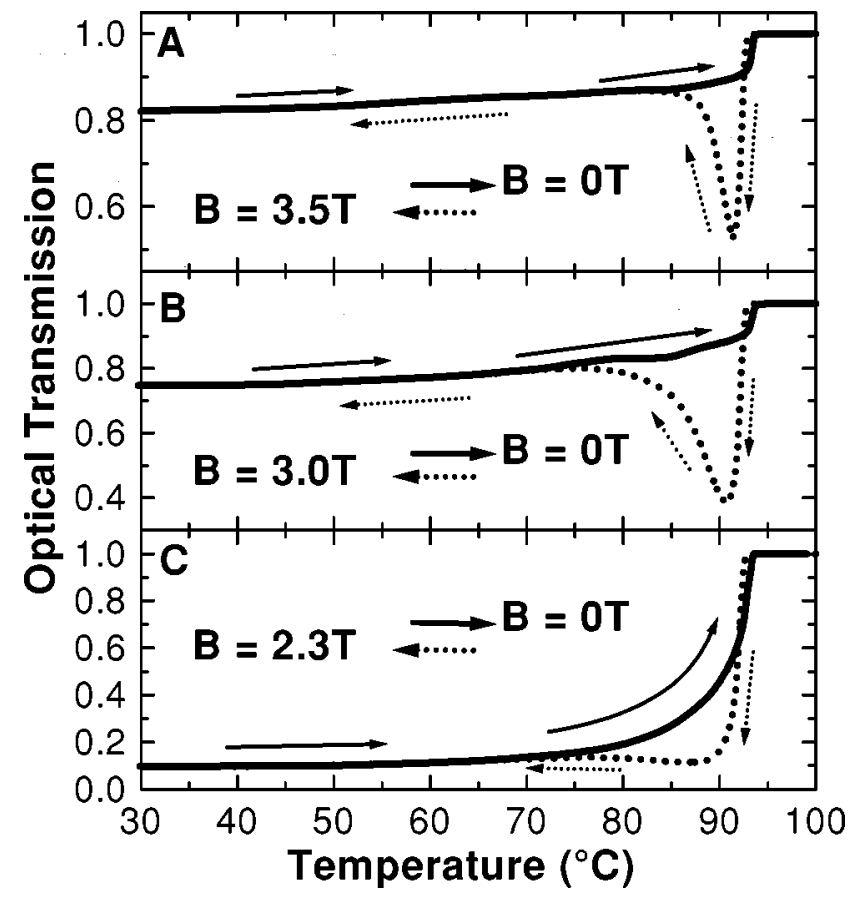

FIG. 4. Optical transmission for a $B 4$ sample with $n=30$, $20 \mu \mathrm{m}$ film recorded during heating up (solid line) in zero field after cooling (dotted line) in a magnetic field of $2.3 \mathrm{~T}(\mathrm{a}), 3.0 \mathrm{~T}(\mathrm{~b})$, and $3.5 \mathrm{~T}(\mathrm{c})$.

nematic region extended from $T_{I-N}$ to $T^{*}$, where $T_{I-N}$ is the LDG result in the absence of fluctuations, Eq. (2). It is visible that via induced alignment the magnetic field reduces the contribution of the fluctuation term from $A_{D}^{2}(B) \sim 0.1$, Ref.
[22], in the absence of any induced order to $A_{D}^{2}(B) \sim 0$ in the case of complete order, narrowing the temperature range of the biphasic region. In the case of high induced ordering the fluctuation contribution tends to zero, the classic LDG result is restored, and the transition is fully first order. When the $I-N$ transition is approached from below, the order fluctuation term does not act anymore as an energetic barrier, with the important consequence that the transition is sharp and there is no biphasic region. Figure 4 presents the optical transmission recorded as the $B 4$ sample approaches the $I-N$ transition from below. In complete agreement with our proposed model, the curves clearly show no biphasic region. The presence of the mesogene-backbone coupling term $K_{m-b} Q$ has the important consequence that once order has been induced in the SCPLCs system, the sample alignment is stable until warmed to the isotropic phase [17]. The different magnetic-field strengths needed to obtain an effect for various SCPCLs compounds can be understood via the fact that structures with a stiffer backbone, shorter spacers or larger polymerization degrees need higher magnetic fields to be aligned at the $I-N$ crossing [17].

We have shown experimentally that a strong magnetic field changes the $I-N$ transition of SCPLCs from a weak first order to a sharp first order via the suppression of the order fluctuations. We propose a qualitative interpretation of this phenomenon within the framework of the LDG theory via the nonlinear coupling of the magnetic field to the order parameter fluctuation. The present results suggest that the mesogene-backbone coupling is coresponsible for the existence of the isotropic-nematic biphasic region, complementary information to the current understanding of the field [4], namely, that only a certain degree of polydispersity accounts for the presence of a biphasic region.
[1] H. Finkelmann, H. Ringsdorf, and J.H. Wendorff, Maclomol. Chem. 179, 273 (1978).

[2] C.B. McArdle, Side Chain Liquid Crystal Polymers (Chapman \& Hall, New York, 1989).

[3] C. Pujolle-Robic and L. Noirez, Nature (London) 409, 167 (2001).

[4] Handbook of Liquid Crystals, edited by D. Demus et al. (Wiley, Weinheim, 1998), Vol. 3, p. 170.

[5] C.S. Hsu, Prog. Polym. Sci. 22, 829 (1997).

[6] J.C. Dubois, P. Lebarny, P. Robin, V. Lemoine, and H. Rajbenbach, Liq. Cryst. 14, 197 (1993).

[7] M. Murakami and H. Fujii, Mol. Cryst. Liq. Cryst. Sci. Technol., Sect. A 225, 259 (1993).

[8] P. van de Witte et al., Jpn. J. Appl. Phys., Part 1 38, 748 (1999)

[9] M. Sawodny, Makromol. Chem. 46, 217 (1991).

[10] V.N. Raja et al., Liq. Cryst. 20, 41 (1996).

[11] R.B. Findlay and A.H. Windle, Mol. Cryst. Liq. Cryst. 206, 55 (1991).

[12] Y. Zhao, P. Roche, and G.X. Yuan, Macromolecules 29, 4619 (1996)

[13] N. Kawatsuki, T. Yamamoto, and H. Ono, Appl. Phys. Lett. 74,
935 (1999).

[14] M. Ungerank, B. Winkler, E. Eder, and F. Stelzer, Macromol. Chem. Phys. 196, 3623 (1995).

[15] M.I. Boamfa, Ph.D. thesis, University of Nijmegen, The Netherlands, 2003, available at http://webdoc.ubn.kun.nl/mono/b/ boamfa_m/index.pdf

[16] Strong and Ultrastrong Magnetic Fields and Their Applications, edited by G. Maret and K. Dransfeld (Springer-Verlag, Berlin, 1985), Chap. 4.

[17] M.I. Boamfa et al., Phys. Rev. Lett. 90, 025501 (2003).

[18] M. Kerker, The Scattering of Light and Other Electromagnetic Radiation (Academic Press, London, 1969).

[19] E.F. Gramsbergen, L. Longa, and W.H. de Jeu, Phys. Rep. 135, 19 (1986).

[20] A.J. Bray, Adv. Phys. 43, 357 (1994).

[21] S. Singh, Phys. Rep. 324, 107 (2000).

[22] Numerically calculated value for director fluctuation, based on Boltzman Statistics. $A_{D}^{2}=\left(\cos ^{2} \theta-\left\langle\cos ^{2} \theta\right\rangle\right)^{2}$, with $\left\langle\cos ^{2} \theta\right\rangle$ $=\int f(\theta) \cos ^{2} \theta \sin \theta d \theta / \int f(\theta) \sin \theta d \theta$. Where $\theta$ describes the director orientation with respect to the magnetic field and $f(\theta)$ is the statistical distribution function describing the director orientation [16]. 